\title{
MDSheet: A Framework for Model-driven Spreadsheet Engineering
}

\author{
Jácome Cunha*, João Paulo Fernandes*† Jorge Mendes*, João Saraiva* \\ *Departamento de Informática, Universidade do Minho, Portugal \\ \{jacome,jpaulo,jorgemendes,jas\}@di.uminho.pt \\ ${ }^{\dagger}$ Departamento de Engenharia Informática, Universidade do Porto, Portugal
}

\begin{abstract}
In this paper, we present MDSHEET, a framework for the embedding, evolution and inference of spreadsheet models. This framework offers a model-driven software development mechanism for spreadsheet users.
\end{abstract}

Keywords-Spreadsheets, Model-Driven Engineering, Software Evolution, Embedded DSLs, Model Inference

\section{INTRODUCTION}

Spreadsheets are widely used by non-professional programmers, the so-called end users, to develop business applications. Spreadsheet systems offer end users a high level of flexibility, making it easier to get started working with them. This freedom, however, comes with a price: spreadsheets are error prone as shown by numerous studies which report that up to $90 \%$ of real-world spreadsheets contain errors [1].

As programming systems, spreadsheets lack the support provided by modern programming languages/environments, like for example, higher-level abstractions and powerful type and modular systems. As a result, they are prone to errors. In order to improve end-users productivity, several techniques have been proposed, which guide end users to safely and correctly edit spreadsheets, like, for example, the use of spreadsheet templates [2], ClassSheets [3], [4], and the inclusion of visual objects to provide editing assistance in spreadsheets. All these approaches propose a form of end user model-driven software development: a spreadsheet business model is defined, from which a customized spreadsheet application is then generated guaranteeing the consistency of the spreadsheet data with the underlying model.

In this paper, we present MDSHEET, a unifying framework where we have integrated the following modelling, manipulation and co-evolution spreadsheet techniques:

- Embedding of ClassSheet models: ClassSheets are a powerful and widely used modelling language to define the business logic of a spreadsheet. MDSHEET embeds this modelling language in a spreadsheet system, providing a coherent environment for model driven spreadsheet engineering, as proposed in [5].

- Co-evolution of ClassSheets and instances: Like any other software artifact, spreadsheets evolve over time. MDSHEET uses a formal setting where the co-evolution of the embedded ClassSheet model and the spreadsheet instance is performed [6].
- Inference of ClassSheets: Being one of the most used programming languages, there are huge amounts of legacy spreadsheets. In order to provide a MDE environment for such legacy spreadsheets, we have implemented in MDSHEET the model inference technique that we have proposed in [3].

In the next three Sections, each of this features is briefly explained. The video that accompanies this paper is also divided in these same parts.

\section{EMbedding ClassSheEts IN SPREAdSheEts}

ClassSheets [4] are a high-level, object-oriented formalism to specify the business logic of spreadsheets. ClassSheets allow users to express business object structures within a spreadsheet using concepts from the Unified Modeling Language (UML). Using the ClassSheets model, it is possible to define spreadsheet tables and to give them names, to define labels for the table's columns, to specify the types of the values such columns may contain and also the way the table expands (e.g., horizontally or vertically).

Besides a textual (and formal) definition, ClassSheets also have a visual representation which very much resembles spreadsheets themselves [7]. We have embedded such visual model representation that mimics the well-known embedding of a domain specific language in a general purpose one. Like in such embeddings, we inherit all the powerful features of the host language: in our case, the powerful interactive interface offered by the (host) spreadsheet system. This approach has two key advantages: first, we do not have to build and maintain a complex interactive tool ${ }^{1}$. Second, we provide Class Sheet model developers the programming environment they are used to: a spreadsheet environment. Furthermore, because the ClassSheet model and the spreadsheet data are defined in the same environment, we now have the power to ensure that the they are synchronized.

In order to illustrate our embedding we present in Figure 1 an embedded ClassSheet model (Figure 1b) and one of its possible instances (Figure 1a).

The ClassSheet model represents pilots, where a pilot is defined by an ID, Name and Flight hours. In row 3, it is defined the type, and the default value, associated with each

\footnotetext{
${ }^{1}$ Like in the Gencel tool, developed by the authors that proposed ClassSheets [8].
} 


\begin{tabular}{|r|l|l|r|}
\hline & \multicolumn{1}{|c|}{ A } & \multicolumn{1}{c|}{ B } & \multicolumn{1}{c|}{ C } \\
\hline 1 & Pilots & & \\
\hline 2 & ID & Name & Flight hours \\
\hline 3 & $\mathrm{pl} 1$ & John & 3400 \\
\hline 4 & $\mathrm{p} 12$ & Mike & 330 \\
\hline 5 & $\mathrm{pl} 3$ & Anne & 433 \\
\hline
\end{tabular}

(a) Pilot table.

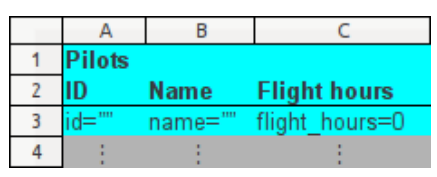

(b) Pilot ClassSheet model.
Figure 1: Pilot example.

column: columns A and B hold strings (with an empty string as default value), and column $C$ holds integer values (with 0 as default). The fourth row of the model contains vertical ellipses in all columns. This means that it is possible for these columns to expand vertically: the tables that conform to this model can have as many rows (entries) as needed.

Reusing the ClassSheet table we built before, we can now model a table to register concrete flights by the airline company, as shown in the right-hand side of Figure 2.

The colors in the model are used to distinguish the different entities represented, namely, pilots, planes, references to pilots in the scheduling table, reference to planes in the scheduling table and the flight scheduling itself.

\section{A. Generating Spreadsheets from ClassSheet Models}

Following the Gencel approach [8], the previously described models can be translated into initial spreadsheets together with tailor-made versions of update operations. These operations are defined to perform the tasks of insertion or deletion in such a way that the spreadsheet correctness is always preserved. In fact, the spreadsheet in the lefthand side of the Figure 2 was produced by our embedding mechanism: the initial spreadsheet will contain the labels in bold on the model, the initial formulas and buttons (grey rows/columns labelled with elipsis) to add new vertical or horizontal blocks of cells.

By using this MDE approach, the end user is guided in the introduction of data that conforms to the underlying model: for example, rows with type integer only accept integer values. Another key feature of this approach is that blocks of cells are automatically produced, for example, to add a new flight, which is a relationship between a pilot and a plane, the user must click on the button in row 6 . The system will then add a new row, also updating the necessary formulas: it will update the formulas in cells $\mathrm{E} 7, \mathrm{I} 7$ and $\mathrm{K} 7$ to include the new added row. This mechanism prevents the user from editing the spreadsheet without correctly updating its formulas, and therefore from corrupting it.

\section{Co-Evolution OF MOdels AND Instances}

One key advantage of using a model-driven software development process is the ability to interact both with the model (a ClassSheet in our case) and its instance (i.e., the spreadsheet data). This is usually a complex task because the model and the instances need to be synchronized! In this section we present a set of co-evolution rules common in spreadsheets. Such rules define evolution steps for ClassSheet models and their instances and they guarantee synchronization. These rules are specified using data refinement theory which provides an algebraic framework for calculating with data types and corresponding values. It consists of type-level coupled with value-level transformations. The type-level transformations deal with the evolution of the model and the value-level transformations deal with the instances of the model (e.g. values).

We have designed an appropriate representation of spreadsheet models, including the fundamental notions of formula and references [6]. For these models and their instances, we have designed coupled transformation rules that cover specific spreadsheet evolution steps, such as the insertion of columns in all occurrences of a repeated block of cells. The rules are divided into three categories [6] combinators, used as helper rules, semantic rules, intended to change the model itself (e.g. add a new column), and layout rules, designed to change the visual arrangement of the spreadsheet (e.g. swap two columns).

Combinator Rules: The first set of rules, combinators, include rules such as after, which means "apply the argument rule after the argument label". These combinators receive a rule as an argument and apply it in a specific place of the model, and thus, they are refinements or isomorphisms if the argument rule is a refinement or an isomorphism.

Semantic Rules: In [6] we have introduced a full catalog of spreadsheet evolution refinement rules. The catalog includes rules such as make it expandable that makes a block of cells expandable (horizontally or vertically), and split that moves a column to a new place and replaces it by references to the new locations. Their full definitions and HASKELL implementations can be found in [6]. As an example, we graphically present in Figure 3 the insert a column rule:

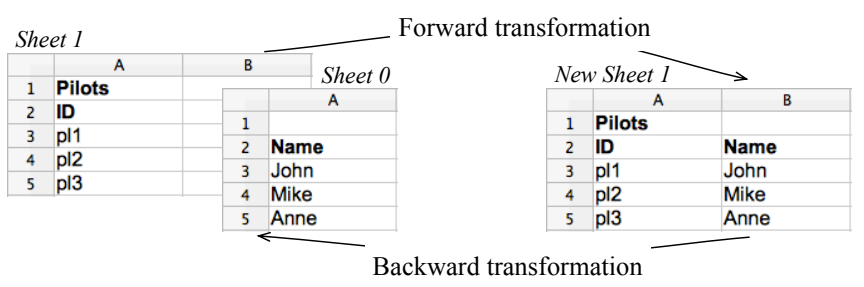

Figure 3: Adding/removing a column visually.

Sheet 1 represents the original spreadsheet with an existing column which is transformed in New Sheet 1 when applied the forward transformation. Applying the backward function, we get the original spreadsheet, Sheet 1 , and a new sheet containing the removed column, Sheet 0 . When applying the forward function Sheet $O$ is not used, and thus, not necessary to exist at that point in time. 


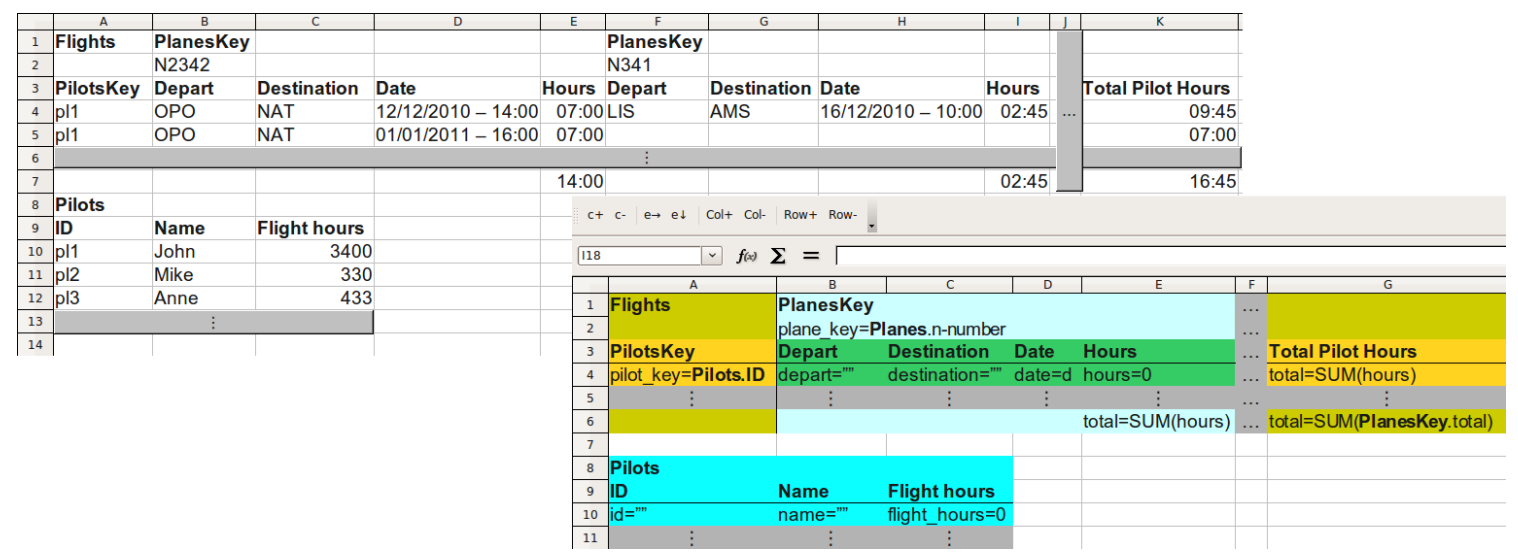

Figure 2: Spreadsheet of an airline company and an abstract model representing it.

The forward transformation, that is, to add a new column is available in the spreadsheet environment as the button $\mathrm{Col}+($ e.g., right-hand part of Figure 2) while the backward function, that is, to remove a column, in the Col-button.

Layout Rules: As the name suggests, layout rules are intended to change the arrangement of spreadsheets only, and not to add or remove any particular information. This set of rules includes evolution steps for changing the orientation of a spreadsheet from vertical to horizontal or to rearrange cells according to some conventions, for example.

Having defined a set of model evolution steps, we can now evolve the model and have the data automatically coevolved. For example, in the ClassSheet of Figure 2, if we wish to add an attribute meal to a flight, we can do it in several steps: first, we add a column named Meal to the Flights class (in the last column of the dark green area, after column E). Second, we set its default value to "NO". Then, these model evolution steps are automatically reflected in the data, with a new column Meal being inserted by the framework in the two Flights instances in the data (new columns are added after column $\mathrm{E}$ and I). Also, the formulas of columns $\mathrm{K}$ are automatically updated.

\section{Classsheet Model Inference}

Consider the example spreadsheet shown in Figure 4. This spreadsheet represents again flights of a company, by in a less organized (but probably more common) manner.

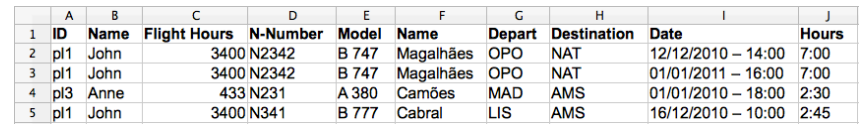

Figure 4: A spreadsheet to store an airline company flights.

The business logic that underlies this spreadsheet is not immediately clear and is quite difficult to infer for a non- expert. In this section we will describe a strategy to infer such a business logic from the data in a spreadsheet.

Objects that are contained in such a spreadsheet and the relationships between them are reflected by the presence of functional dependencies between spreadsheet columns.

It is possible to construct a relational model from a set of observed functional dependencies [3]. Such a model consists of a set of relation schemas (each given by a set of column names) and expresses the basic business model present in the spreadsheet. Each relation schema of such a model results from grouping functional dependencies together. For example, for the spreadsheet in Figure 4 we can infer the following relational model (underlined columns indicate those on which the other columns are functionally dependent (primary keys); \# indicates attributes referencing other table columns (foreign keys); tables between symbols $<>$ represent relationship):

Pilots (ID, Name, Flight hours)

Planes (N-Number, Model, Name)

$<$ Flights $>$ (\#ID, \#N-Number, Depart, Destination, Date, Hours)

The model has two relations, Pilots and Planes, and a relationship connecting them, Flights. A relational model is very expressive, but it is not quite suitable for spreadsheets From this relation model a Class Sheet can be generated, as explained in [3]. In fact, the model generated is the one shown in Figure 2.

The architecture of our approach is sketched in Figure 5.

\section{Tool Architecture}

In this section we present the architecture of our modeldriven spreadsheet environment. In this environment, end users can interact both with the ClassSheet model and the spreadsheet data. Our techniques guarantee the synchronization of the two representations. In this setting, the spreadsheets consists of two sheets: Sheet 0 , containing the embedded Class Sheet model and Sheet 1, containing the spreadsheet data that conforms to the model. We have 


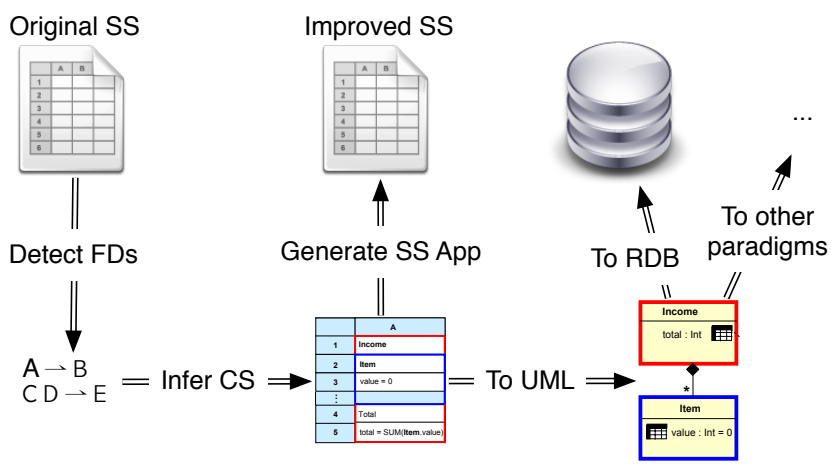

Figure 5: Overview of the process of inferring a ClassSheet.

defined an add-on to a widely used spreadsheet system, the OpenOffice.org system, so end users can evolve their models by using predefined buttons in the spreadsheet environment For each button, we defined a OpenOffice.org BASIC script that interprets the desired functionality, and send the contents of the spreadsheet (both the model and the data) to the MDSHEET framework. The MDSHEET framework was developed in HASKELL, and implements the co-evolution of the spreadsheet models and data. The global architecture of the tool we developed is presented in Figure 6.

In this environment end users can build the ClassSheet from scratch using the provided buttons. However, we consider also the inference of the model from the spreadsheet data [3]. This is particularly important when we are considering legacy spreadsheets. Moreover, the generated refactored spreadsheet includes some business logic rules (expressed as spreadsheet formulas) that assist end users in the safe and correct introduction/editing of data.

Tool and demonstration video availability: The MDSHEET tool and a video with a demonstration of its capabilities are available at the SSaaPP project web page: http://ssaapp.di.uminho.pt/mediawiki/index.php/Software.

\section{REFERENCES}

[1] R. Panko, "Spreadsheet errors: What we know. what we think we can do." Proceedings of the Spreadsheet Risk Symposium, European Spreadsheet Risks Interest Group (EuSpRIG), 2000.

[2] R. Abraham and M. Erwig, "Inferring templates from spreadsheets," in Proc. of the 28th Int. Conf. on Software Engineering. New York, NY, USA: ACM, 2006, pp. 182-191.

[3] J. Cunha, M. Erwig, and J. Saraiva, "Automatically inferring classsheet models from spreadsheets," in 2010 IEEE Symposium on Visual Languages and Human-Centric Computing. IEEE Computer Society, 2010, pp. 93-100.

[4] G. Engels and M. Erwig, "ClassSheets: automatic generation of spreadsheet applications from object-oriented specifications," in 20th IEEE/ACM Int. Conf. on Automated Sof. Eng., Long Beach, USA. ACM, 2005, pp. 124-133.

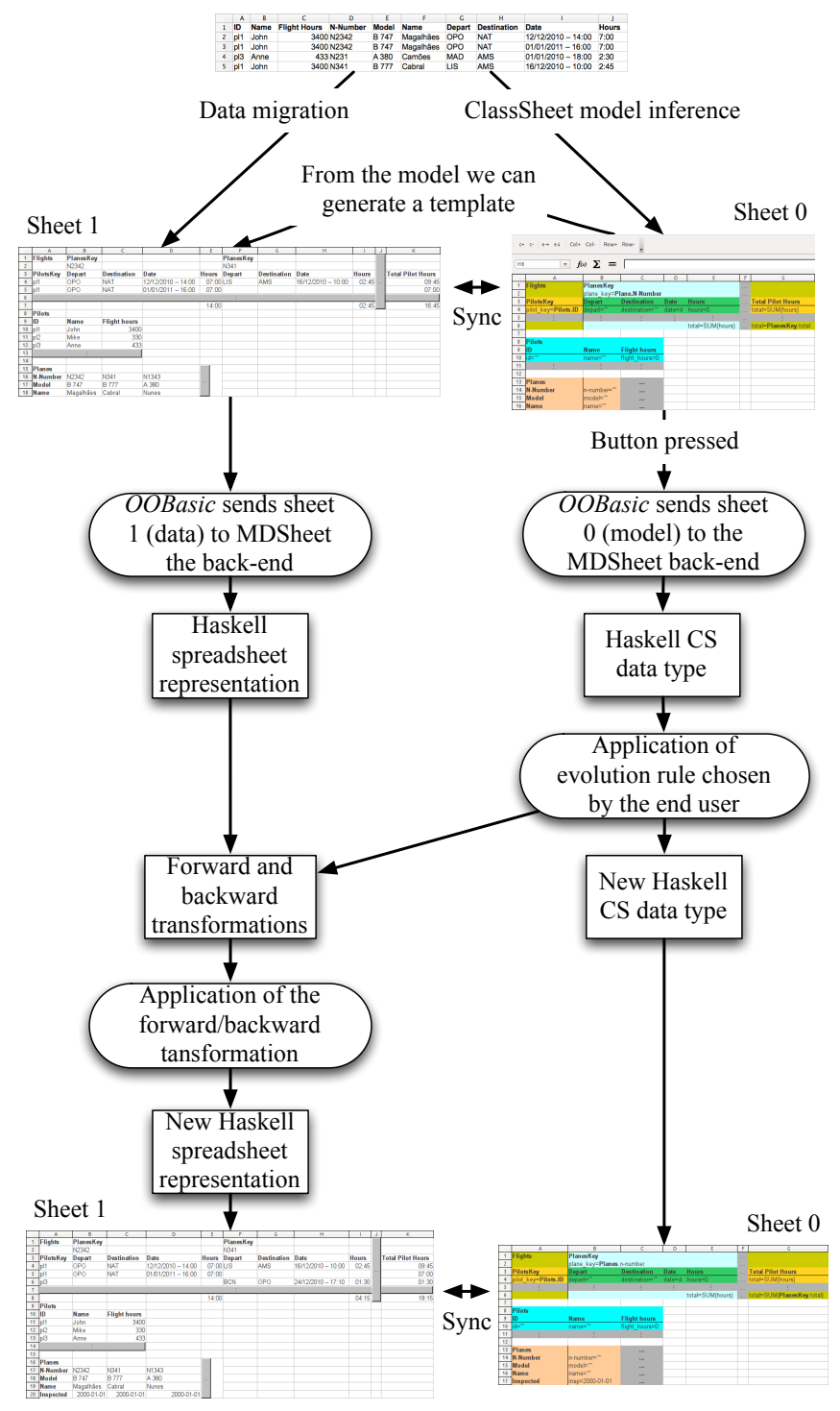

Figure 6: Spreadsheet model-driven environment.

[5] J. Cunha, J. Mendes, J. P. Fernandes, and J. Saraiva, "Embedding and evolution of spreadsheet models in spreadsheet systems," in IEEE Symp. on Visual Languages and HumanCentric Computing. IEEE CS, 2011, pp. 186-201.

[6] J. Cunha, J. Visser, T. Alves, and J. Saraiva, "Type-safe evolution of spreadsheets," in Int. Conf. on Fundamental Approaches to Software Engineering, ser. FASE'11/ETAPS'11. Berlin, Heidelberg: Springer-Verlag, 2011, pp. 186-201.

[7] M. Erwig, R. Abraham, I. Cooperstein, and S. Kollmansberger, "Automatic generation and maintenance of correct spreadsheets," in Proc. of the 27th Int. Conf. on Software Eng. New York, NY, USA: ACM, 2005, pp. 136-145.

[8] R. Abraham, M. Erwig, S. Kollmansberger, and E. Seifert, "Visual specifications of correct spreadsheets," in IEEE Symposium on Visual Languages and Human-Centric Computing. IEEE Computer Society, 2005, pp. 189-196. 